\title{
The Automatic Nerve Signal Transmission and Conscious Primacy Hypothesis
}

\author{
Zhiyin Shan \\ Doublegalaxy Technologies, Tustin, California, USA \\ Email:Zhiyinw@yahoo.com
}

How to cite this paper: Shan, Z. (2017) The Automatic Nerve Signal Transmission and Conscious Primacy Hypothesis. Journal of Behavioral and Brain Science, 7, 165-179.

https://doi.org/10.4236/jbbs.2017.74014

Received: March 6, 2017

Accepted: April 10, 2017

Published: April 13, 2017

Copyright (C) 2017 by author and Scientific Research Publishing Inc. This work is licensed under the Creative Commons Attribution International License (CC BY 4.0).

http://creativecommons.org/licenses/by/4.0/ (c) (†) Open Access

\begin{abstract}
This paper proposes a hypothesis about automatic nerve signal transmission and conscious primacy. If stimulated suddenly, people are initially unaware of the stimulation. Without conscious guidance, nerve signals are automatically transmitted via the strongest neural connections, thereby producing fast and involuntary behavior. Upon awareness of the stimulation, conscious primacy plays a role, and nerve signals are transmitted in consciously guided directions, thereby generating cognition and voluntary behaviors. When we repeat an action in a special environment, it will become a habit. This is because when nerve signals are repeatedly transmitted to action cells in a specific environment, neural connections between the specific environment and the action are strengthened. Upon return to this specific environment, nerve signals will be automatically transmitted, producing a stereotyped habit behavior without conscious thought. Facial expression, for example, is controlled by both emotion and cognition. Prior to emotional awareness of sudden stimulation, nerve signals are automatically transmitted, producing microexpressions consistent with emotion. Upon awareness, control switches from emotion to cognition, producing flexible facial expressions consistent with consciousness. The automatic nerve signal transmission and conscious primacy hypothesis suggests a new mechanism for producing different behaviors, reveals the essential difference and mutual transformation of stereotyped and flexible behaviors, and opens a new field for ethological study.
\end{abstract}

\section{Keywords}

Behavior, Neural Connection, Consciousness, Unconsciousness, Synapse

\section{Introduction}

People generally believe that decisions they make and the corresponding actions they perform are consciously initiated and controlled. However, there is strong 
emerging evidence that unconscious processes influence decision-making [1] [2] [3], and that automatic behaviors can be unconsciously elicited [4] [5].

Libet [1] demonstrated that the onset of cerebral activity, termed the readiness potential, occurs at least several hundred milliseconds before conscious intentions to act; therefore, neural activity starts automatically before conscious intentions to act. A functional magnetic resonance imaging (fMRI) study found brain activity before decisions reached awareness [6]. Furthermore, a study detecting neural activity using implanted electrodes found that neurons were activated at least several hundred milliseconds before awareness of a decision to act [7]. Visual stimuli can unconsciously result in motor responses [4]. For example, patient P.S. has a left homonymous hemianopia resulting from right cerebral damage, and left-sided neglect persists despite free movement of her head and eyes. She reports that she cannot see anything on her left side. P.S. was presented with two pictures of houses, which were placed on a desk in front of her and centered at the midsagittal plane of her head. In one of the two pictures, the house on the left was on fire, while the house on the right was not on fire. She was asked to distinguish the difference between the houses, and she judged that the houses were identical. However, when asked to select the house that she would prefer to live in, she selected the house that was not burning in nine of 11 trials. P.S. did not consciously see that the house was burning in the first scenario, but she did make appropriate selection decisions. Therefore, the burning characteristics were processed unconsciously. Next, two new pictures, one with flames on the right side of the house, and one house that was not on fire, were presented simultaneously. When P.S. was again asked which house she preferred, she immediately noticed the flames, and chose the non-burning house in six of six trials.

In this paper, we present the automatic nerve signal transmission and conscious primacy hypothesis. We first discuss the basic neurobiological mechanisms of automatic unconscious nerve signal transmission. Then, we discuss our hypothesis in the context of experimental findings and use it to explain the generation of psychological phenomena including habits, dreams, and cocktail party effect.

\section{The Content of Automatic Nerve Signal Transmission and Conscious Primacy}

Automatic nerve signal transmission and conscious primacy occurs when sensory receptors convert stimuli into nerve signals, which transmit automatically according to the strongest neural connections in the neural network. If nerve signals reach motor neurons or effector neurons, instinctive and unconscious behavior is produced. However, under the control of consciousness, the brain may analyze the stimuli and change the direction of nerve signal transmission, thereby resulting in nerve signals transmitting to other motor neurons and producing conscious or voluntary behavior. Therefore, automatic nerve signal transmission occurs first. Upon awareness of the stimulation, conscious primacy plays a role. The change from automatic transmission to conscious transmission 
may then block instinctive and unconscious behaviors, which are replaced by conscious voluntary behaviors. However, if conscious intervention does not occur prior to automatic signal processing, then automatic nerve signal transmission will produce instinctive and unconscious behaviors.

\section{The Basic Neurobiological Mechanisms of Nerve Signal Transmission}

\subsection{Nerve Signals Transmit Automatically without Consciousness}

Electrophysiological experiments are one of the most fundamental types of neuroscience experiments, in which responses are generated by electron stimulation without consciousness. In the 18th century, Luigi Galvani found that a dead frog's leg kicked as if attached to a living being when the nerve was stimulated by electricity [8]. Penfield and Boldrey applied electrical stimulation to the cortical surface of their patients' brains during neurosurgery, finding that corresponding muscles were activated, and even produced simple movements. In 1937, they mapped the motor homunculus [9]. Nobel Prize winner Eric Kandel stimulated abdominal ganglion cells of aplysia with a microelectrode and produced powerful gill contractions. This was an exciting finding, as it was the first identification of a motor neuron that controlled a specific behavior [10]. Based on the above phenomena, these muscle movements were generated by exogenous electrical stimuli rather than by the brain, indicating that nerve signals have the capacity to transmit to muscles and produce behavior, even without conscious control.

\subsection{Nerve Signals Are Transmitted via Action Potentials, Which Are Generated Depending on Integrated Postsynaptic Potentials}

Nerve signals are transmitted by axons via action potentials, which occur following integrated postsynaptic potentials. The integrate-and-fire neuronal model is one of the most widely used models for analyzing behavior in neural systems [11] [12]. The spread of postsynaptic potentials rapidly decreases, therefore, it is impossible to conduct the signal from the synapse to the axon hillock; several postsynaptic potentials must integrate to form a larger potential and trigger an action potential.

\subsection{Nerve Signals Transmit between Neurons via Synapses}

Nerve signals transmit between neurons via synapses, which are the structure connecting two neurons. When electrical nerve signals reach the synapse, the presynaptic membrane releases neurotransmitters into the gap, which then bind to specific receptors on the postsynaptic membrane and generate postsynaptic potentials. The postsynaptic potentials combine into an overall signal by integrating in space and time. When integrated signals reach a threshold at the axon hillock, an action potential is generated and the signal can be conducted to other neurons.

The spatial distribution of synapses varies across the neuronal body and den- 
drites, and presynaptic neurons also release neurotransmitters at different times. Additionally, there are both excitatory and inhibitory synapses on a single neuron. Overall, postsynaptic potentials are integrated over space and time, as well as by the combination of excitatory and inhibitory synapses, to achieve postsynaptic potentials that exceed the firing threshold and generate action potentials [13] [14] [15].

\subsection{All-or-None Properties of Action Potentials Create Neuronal Switches}

The action potential is an all-or-none response; it is either on or off, like a light switch. When the integrated postsynaptic potential is below the firing threshold, an action potential will not occur at all, but when the integrated postsynaptic potential exceeds the firing threshold, an action potential will occur, regardless of stimulus intensity. If the threshold is not reached, the action potential cannot be generated, and nerve signal transmission is temporarily blocked until the postsynaptic potential reaches the threshold.

\subsection{Nerve Signals Automatically Transmit Based on the Strongest Neural Connections}

When nerve signals easily transmit between neurons via synapses, it is termed a strong neural connection; when nerve signals do not easily transmit to other neurons, it is termed a weak neural connection. One neuron typically has synaptic connections with multiple neurons; therefore, activation of one neuron can potentially propagate nerve signals to numerous connecting neurons. When the activated neuron has multiple strong neural connections, the signals will transmit via many pathways; however, when the activated neuron has strong neural connections with only one neuron, the nerve signal will only be transmitted via one pathway. If all neural connections to the activated neuron are weak, signal transmission will be terminated.

\subsection{Neural Connections Are Plastic, and Both Learning and Training Can Increase Their Strength}

Hebb proposed the following hypothesis regarding neural connections: "When an axon of cell $A$ is near enough to excite a cell $B$ and repeatedly or persistently takes part in firing it, some growth process or metabolic change takes place in one or both cells such that $A$ 's efficiency, as one of the cells firing $B$, is increased" [16]. The automatic nerve signal transmission and conscious primacy hypothesis relies on the principles of neural connection strength and action potential generation. Any changes, such those to distribution, positions of synapses, generation time of postsynaptic potentials, and ratio of excitatory and inhibitory postsynaptic potentials, will affect the strength of neural connections. Synapses are the structural basis of neural connections, therefore, increasing synaptic strength produces strengthened neural connections. Synaptic strength can be changed by learning and environmental exposure [17] [18]. For example, increases in synap- 
tic strength are observed in rats exposed to environmental enrichment [18]. One study housed rats in a room filled with boxes, wooden ramps, and other surfaces for them to run on. The rats were then chronically implanted with stimulating and recording electrodes in the perforant path and the ipsilateral dentate gyrus, respectively. Significant increases in both excitatory postsynaptic potentials (EPSPs) and voltage spike magnitudes occurred with environmental enrichment $\left(t_{\mathrm{ESPS}}=38.09, \mathrm{P}<0.005 ; t_{\text {spike }}=32.5, \mathrm{p}<0.005\right)$ [19]. In another motor skills training experiment, rats trained to traverse a complex series of obstacles possessed significantly more synapses per Purkinje cell than rats that traversed an obstacle-free runway. Therefore, learning and training increased synaptic strength under these conditions [20].

\section{Stimulus-Generated Automatic Nerve Signal Transmission in Unconscious States}

There is a time lapse between being stimulated and being aware of stimulation, and conscious decisions occur after both components have occurred. Behavior cannot be guided by consciousness in the interval between these two events, but cerebral potential changes occur in the brain. The potential changes during this period of time are termed the readiness potential [1]. The readiness potential theory proposes that the onset of readiness potential precedes awareness of intending to act by several hundred milliseconds; brain activity has already been initiated before there is any subjective awareness of a "decision" to act.

Unconscious movement has been observed in a visual search experiment [5]. Observers were instructed to make a voluntary goal-directed saccade to a target. During the experiment, both the target and a new object were presented simultaneously. Eye movements were recorded using an eye link tracker, and observers were asked whether they were aware of the effects of a new object. The recordings demonstrated that the observers' eyes went in the direction of the new object, stopped briefly ( $25-150 \mathrm{~ms}$ ), and then returned to the original target. However, the observers believed that their saccades were not affected by the new object. These results indicate that the observers were not aware of their eye actions, despite that eye movements in the direction of the new object were clearly recorded by the tracking equipment. Furthermore, the design of this visual search required that the observers must move their eyes to the targets as soon as doing so was under conscious control. Therefore, unconscious eye movements in the direction of the new object were automatic.

Automatic nerve signal transmission and conscious primacy hypothesis explains that the original target and new object stimuli were received by the eyes and converted to nerve signals, which traveled automatically to the motor neurons and generated eye movements. When observers became aware of the target stimuli, the brain began to analyze target and instruction information, in order to make conscious decisions. Because of readiness potentials, at the initial stages without conscious control, nerve signals automatically generate eye movements toward the new object based on strong connections for a new object. After the 
readiness potential period elapses and observers are aware of the target, they follow instructions to make a voluntary goal-directed saccade to the target. This goal-directed event is accomplished by initiation of nerve signals that underlie the action of eye movement to the target. Therefore, observers were aware of seeing the target, but did not know that their eyes had moved to the new object.

Priming is a brief stimulus presentation that does not reach conscious awareness, but which can affect motor responses to subsequently presented target stimuli. In priming experiments, arrows are often used as primer and target stimuli [21] [22]. In one such experiment, trials started with a brief presentation of a primer stimulus, followed by a mask and a target stimulus. Participants were instructed to point in the direction of target arrows, using their left or right hands, and to respond as soon as possible. Trials were termed compatible when primer and target arrows were pointing in the same direction, and incompatible when primer and target arrows were pointing in different directions. Responses are typically faster and more accurate in compatible than incompatible trials. The duration of primer exposure was too short to be noticed by participants; however, compatible primers increased the response speed.

According to the automatic nerve signal transmission hypothesis, when left-pointing primer arrows are presented, the stimulus is received by the eye and converted into a nerve signal that automatically travels in the left direction, because the participants are not aware of the stimulus and signal transmission is therefore not under conscious control. Additionally, when a left-pointing target arrow is presented, participants become aware of the stimulus from the left side, and voluntarily point their hands to the left. Because the directions of both the primer and the target are the same, the directions of automatic transmission and conscious transmission overlap, thereby accelerating the decision-making speed via compatible effects. In contrast, when the primer arrow points to the left and the target arrow points to the right, the direction of automatic transmission is left and conscious transmission is right; therefore, opposite directions reduce processing speed as a result of incompatible effects.

\section{Consciousness-Guided Nerve Signal Transmission in Conscious States}

Consciousness enables human behavior to be flexible. However, the mechanisms of consciousness are not clear. For the present discussion, consciousness is defined as an ability to perceive stimuli and make decisions by analysis, which results in strengthened or changed behavior. Conscious primacy results in nerve signals being transmitted according to conscious guidance, thereby resulting in cognition and voluntary behavior.

In a study of free will, participants responded to two stimulus signals [23]. The first stimulus signal was a button press, where participants were instructed to press the button as soon as possible following stimulus presentation. The button press comprised $75 \%$ of trials. The second stimulus signal was varied between two different presentations. One presentation was a stop signal; upon 
seeing this signal, participants were instructed to stop pressing the button immediately, without thinking about it. The other presentation was a decide signal; upon seeing this signal, participants decided whether to stop or continue pressing the button. In the button press trial, the peak reaction time was $700 \mathrm{~ms}$; in the stop trial, the peak reaction time was $600 \mathrm{~ms}$; in the decide trial, there were two peaks, one at $600 \mathrm{~ms}$ and the other at $1300 \mathrm{~ms}$. When the stop signal appeared after the button press signal, participants were instructed to immediately stop pressing the button, however, some participants continued to press the button, and the peak was $100 \mathrm{~ms}$ earlier at $600 \mathrm{~ms}$, which may have resulted from actions that started early. If button pressing started early, participants may not have enough time to cancel the button press. In decide trials, the decide signal appeared after the button press signal, and participants could decide to continue pressing the button or stop pressing the button, therefore the peak would be delayed and the results consequently had two peaks.

The automatic nerve signal transmission and conscious primacy hypothesis can explain the different peaks observed by Kühn and Brass [23]. In 75\% of the trials, only one signal appeared, and when participants pressed the button as soon as possible without conscious processing, they obtained the best score. Therefore, the button pressing action became a habit that did not require conscious control. Based on the automatic nerve signal transmission hypothesis, if nerve signal transmission occurs without conscious control, it is automatic transmission. When the stop signal appears, one of two results may occur: 1) the stop signal is received in time, the action of pressing the button is terminated, and nothing is recorded, or 2) the stop signal is received too late to cancel the button, the action of pressing the button action cannot be terminated, therefore this response is recorded. This recorded action is produced following the button press signal, which suggests that in stop trials, the button pressing results from automatic nerve signal transmission. In contrast, when a decide signal appeared, time was required for conscious decision-making. In this scenario, there were also two results: 1) when participants decided to stop pressing the button, nothing was recorded, and 2) when participants decided to press the button, two peaks were recorded, at $600 \mathrm{~ms}$ and $1300 \mathrm{~ms}$. Free decision-making depends on consciousness. According to the principle of conscious primacy, once consciousness is active, nerve signals will transmit according to conscious guidance. However, this conscious decision-making process takes time, so the peak appeared at $1300 \mathrm{~ms}$. To explain the peak at $600 \mathrm{~ms}$, we propose that this peak was similar to the peak both in the button pressing trials and the stop trials, and occurred following automatic transmission. When participants received a decision signal, some of the button pressing actions were almost finished and there was not enough time to change responses, so the button was pressed at about $600 \mathrm{~ms}$. Therefore, the first peak resulted from automatic nerve signal transmission, and the second peak resulted from consciousness, as a component of free decisionmaking. 
Facial expressions reflect emotions. For example, people generally laugh when they are happy, and cry when they are sad. However, facial expressions do not always express actual feelings. For example, upon encountering a person who is disliked, people may smile and greet each other to be polite instead of displaying disgust; this interaction is the result of cognition. Affect and cognition are highly interdependent, but the contributions of affective primacy vs. cognitive primacy have been debated for several decades [24] [25].

Emotions can also rapidly evoke facial responses in the absence of conscious awareness [26] [27]. Dimberg et al. [28] conducted an experiment to investigate whether facial reactions can be unconsciously elicited. Participants received $30 \mathrm{~ms}$ exposures to happy, neutral, and angry target faces, which were immediately followed and masked by neutral faces for $5 \mathrm{~s}$. Target faces were only presented for $30 \mathrm{~ms}$, which is a duration that does not enable conscious processing. None of the participants reported that they had seen the target faces. Facial EMG activity in the zygomatic major and corrugator supercilii muscle regions was measured, with results indicating that facial expressions evoked by emotions are rapid and unconscious.

In daily life, people often have facial expressions that are consciously controlled but inconsistent with their underlying emotions. Prior to inconsistent facial expressions, there are subtle changes in expression, known as microexpressions. Microexpressions are brief facial expression for 1/25 - 1/5 s, and are evoked unconsciously by stimuli. Facial muscle activity that is consistent with the true emotion can be recorded during microexpressions. Microexpressions are also consistent with experimental findings that facial expressions are rapid and unconscious. However, the mechanisms for rapid changes to inconsistent facial expressions have not been elucidated. The automatic nerve signal transmission and conscious primacy hypothesis can explain the switch between two expressions. During the response to a facial expression eliciting stimulus, nerve signals automatically transmit to facial muscles and generate facial expressions rapidly and unconsciously. There is an initial reading potential period, during which the participant is not aware of the stimulus. Once the participant is aware of the emotion, the brain begins to be controlled by consciousness, at which time the conscious primacy principle initiates a change in nerve signal transmission. Under the guidance of consciousness, nerve signals for facial muscle movements are generated, thereby producing facial expressions consistent with cognition. The automatic nerve signal transmission and conscious primacy hypothesis suggests that during responses to stimuli, the facial expression consistent with the true emotion appears rapidly and involuntary at first, followed by a switch to facial expression consistent with cognition.

This rapid switching can be described using the example of a salesman who carefully prepared a presentation of new products. He thought that the presentation would be successful; however, after the presentation, the boss had opinions that indicated some dissatisfaction. Upon hearing these opinions, an unhappy expression appeared on the salesman's face, but this expression switched to a 
happy expression almost immediately, before being noticeable to others. Common sense would indicate that the salesman should welcome his boss's opinions, so he appears to be happy. However, the opinions are negative stimuli to the salesman, which are automatically processed by neural networks for unhappy emotions. The salesman cannot immediately be aware of his unhappy expression, but becomes aware of it only after the reading potential, at which point he can use cognition to switch to a happy expression. The voluntary reaction that results from cognition is slower than the automatic reaction to stimuli.

\section{Automatic Nerve Signal Transmission Explains Behavior and Psychological Phenomena}

\subsection{Habits}

William James wrote in the book The Principles of Psychology, "When we look at living creatures from an outward point of view, one of the first things that strike us is that they are bundles of habits. It thus appears that habit covers a very large part of life." Similarly to other animals, humans can repeat the same actions in the same environment, thereby forming habits and the associated actions. Examples include washing one's face and brushing one's teeth automatically after waking up every morning or always going home along the same route after work. These habits develop unconsciously, and it is possible to perform many actions automatically, without conscious control [29]. The automatic nerve signal transmission and conscious primacy hypothesis proposes that when the same actions are repeated many times in a particular environment, the neural connections are strengthened and produce networks between the neurons receiving stimuli and producing actions. In the same environment, the same stimulations are received and converted into nerve signals that conduct automatically via the pathway that has the strongest neural connections, thereby inducing the habit action.

It is difficult to change habits that are formed in particular environments, and changing such habits requires intense determination. Automatic nerve signal transmission allows actions to occur automatically, thereby eliminating the need for thinking and decision-making. To overcome negative habits, the pathways of nerve signal transmission must be changed by conscious efforts, and therefore require conscious thought and intention. Under these circumstances, there is resistance to change, therefore, a person must be very determined to overcome the resistance. Tam, Bagozzi, and Spanjol [30] found that participants with unhealthy snacking habits consumed more healthy snacks when forming implementation intentions. In another experiment, researchers observed the effects of conscious decisions regarding fruit and vegetable consumption. Group A planned to eat fruit and vegetables, while group B not only planned to eat fruit and vegetables, but also practiced overcoming motivational barriers to eating fruit and vegetables. The results demonstrated that both groups increased fruit and vegetable consumption, but group B had a larger increase in consumption [31]. 


\subsection{Dreams}

The automatic nerve signal transmission hypothesis proposes that the brain is in a state of unconsciousness during sleep; but that nerve impulses continue to be transmitted in the cortex. One function of these nerve impulses is posited to be automatic transmission to retrieve memories, which contribute to dreams.

Freud used his psychoanalytic theory to analyze dreams and proposed that dreams are fulfillments of wishes or expressions of impulses that are repressed during awake states. He interpreted the implied meanings of dreams and used these interpretations to treat patients with psychological disorders. While psychoanalysis has many supporters, there are also many people who dispute its validity [32] [33]. The automatic nerve signal transmission hypothesis proposes that during unconscious sleep states, lack of awareness of environmental stimuli means that information cannot be analyzed to contribute to decision-making. However, during sleep, nerve signals continue to transmit in the cortex without conscious control in accordance with the strongest neural connections. People aspire to achieve wishes and desires. The neural connections underlying wishes are therefore among the strongest connections. According to the conscious primacy hypothesis, wishes are suppressed and resisted by the conscious mind during awake hours. For example, upon seeing a beautiful flower at a park that cannot be picked during the day, a person who knows the park rules may enjoy the flower by looking at it instead. During dreaming, when conscious control does not occur, it is possible to pick the flower. When nerve signals transmit information for a wish with the strongest neural connections, the wish appears in the dream and fulfills the task that was resisted during the day.

The contents of dreams are derived from memory, but dreams often alternate between multiple episodic memories [34], which are difficult to explain using psychoanalysis. This is one reason for objections to psychoanalysis. The automatic nerve signal transmission hypothesis can account for alternation between episodic memories during dreaming because nerve impulses can be transmitted from one neuron to multiple neurons, including multiple neural pathways. When neural signals transmit primarily via one pathway, this is termed the main pathway, and others are termed side pathways. There are usually many side pathways associated with a main neural pathway. When awake, nerve signal transmission is guided by consciousness (Figure 1), but during sleep (without conscious control), neural signals transmit along the strongest neural connections, including side pathways (Figure 2). Therefore, the plots of dreams constantly alternate between multiple neural pathways, resulting in discontinuous dream contents.

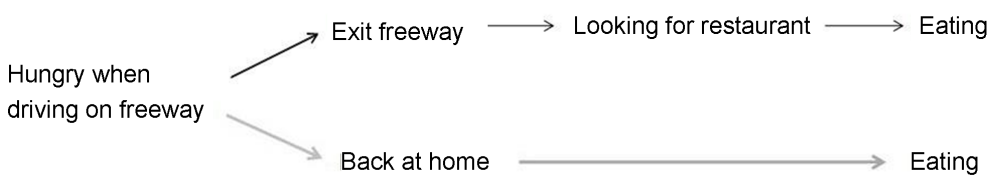

Figure 1. During wakefulness, nerve signal transmission is guided by consciousness. 
A woman who prefers eating at home is driving on a freeway to Las Vegas. If she feels hungry, she will exit the freeway to find a restaurant instead of driving back home to eat. Although the neural connections between being hungry and eating at home are stronger than those between being hungry and eating at a restaurant, her consciousness forces nerve signals to transmit along pathways for exiting the freeway to find a restaurant instead of returning home to eat.

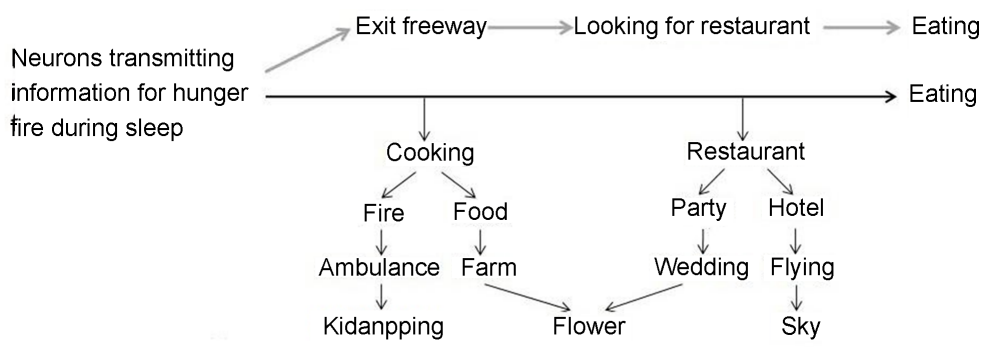

Figure 2. During sleep, nerve signals transmit according to the strongest neural connections.

In the evening, while the woman is sleeping at a hotel in Las Vegas, the neurons that transmit information for hunger fire in her cerebrum, and the nerve signal will be transmitted via the main pathway for eating instead of for exiting the freeway because the connection between hunger and eating is very strong. In addition to the main pathway for eating, there are many side pathways. The nerve signals may therefore be transmitted along the strongest neural connections, which may be from cooking to fire to ambulance to kidnapping, or from cooking to food to farm to flower. If the woman does not like cooking, the nerve signals may be transmitted to restaurant, then from restaurant to hotel to flying to sky, or from restaurant to party to wedding to flower. Therefore, in the dream, the woman may dream of concepts related to cooking, including food, farm, flower, fire, ambulance, and kidnapping, or her dream may include restaurant, party, wedding, hotel, flying, and sky. This example explains how the plots of dreams shift constantly and discontinuously.

\subsection{Cocktail Party Effect}

The cocktail party effect refers to the ability to focus one's attention on a particular person's voice amongst other voices and background noise. A common experience, such as while reading an interesting book, is that a person may not notice the content of conversation between people nearby. However, if someone mentions the person's name, attention will shift to process this information. Experiments have demonstrated brain responses to a person's own name while sleeping, even without conscious awareness of the name being presented [35] [36].

The automatic nerve signal transmission hypothesis may explain why irrelevant topics can be ignored but processing can quickly shift when the information becomes relevant. When reading an interesting book with people speaking nearby, the story attracts the person, making him focus on its contents. 
When a person is reading an interesting book and immersed in the story, even if there are other people talking nearby, he is still able to focus on its contents. The speaking voice, which is irrelevant, will pass through his ear and be transmitted by his brain, but the neural connections are very weak and the signal transmission will be terminated. The signal is therefore not consciously heard. A person's own name is repeated by others every day, so this information is frequently transmitted by the neural networks and the neural connection is fortified. When one's own name is mentioned while reading, the name impulse will automatically conduct to the associated neurons and attract conscious attention.

\section{Future Research Aspects}

The automatic nerve signal transmission and conscious primacy hypothesis should be verified by experimental testing of associated hypotheses. If this hypothesis is supported, there may be extensive applications across scientific fields.

\subsection{Biological Science}

Is correct memory retrieval related to the strength of neural connections? It is clear that neural connections are plastic. Can the direction of memory retrieval be changed when neural connection strength is increased or decreased, thereby creating false memories?

Many antipsychotic drugs can reduce patient's symptoms, but when patients stop taking the medicine, the symptoms come back. Does this mean that schizophrenia is related to strong but inappropriate neural connections? Is it possible that a false memory can be extracted, producing hallucinations, when nerve signals transmit automatically along a strong but inappropriate neural pathway? Maybe antipsychotic drugs inhibit the nerve signals' transmission, temporarily bringing symptoms under control, but if the patient stops taking antipsychotic drugs, the symptoms reappear.

Is Alzheimer's disease related to neural connections? Maybe as neural connections become weak, nerve signal transmission speed is reduced, slowing cognition. The connections become increasingly weak until they are damaged, resulting in an inability to extract the memory and dementia.

Is addiction related to neural connections? Can automatic transmission be used for information processing in artificial intelligence?

\subsection{Social Science}

Are neural connections related to the mind? After birth, children receive stimulation from the external environment, and new neural connections constantly form and develop. By aiding mental maturation, a good social environment helps children achieve healthy mental growth.

Why do people's cognition and behavior vary by environment? Maybe in different environments, people receive different stimuli that change neural connections, so the nerve signal transmission direction changes. This in turn changes cognition and behavior, so a good social environment is also important for 
adults.

Offensive language will strengthen people's neural connections on associated pathways. If automatic nerve signal transmission has undesirable consequences, who should be held responsible? How can automatic nerve signal transmission on the associated pathways be avoided? What are the implications for freedom of speech?

\subsection{Daily Life}

Automatic nerve signal transmission produces good and bad habits. To overcome bad habits, the direction of nerve signal transmission must be consciously changed. Can bad habits be easily overcome by conscious effort? Is a strong will needed? Based on the mechanism of habit formation, how can good habits be maintained and bad habits be prevented?

Maybe someone who has obsessive-compulsive disorder has strong inappropriate neural connections resulting from a strong suggestion or experience. If automatic transmission on inappropriate neural connections can cause disorders, avoiding inappropriate neural connections and weakening existing ones will be very important.

\section{Conclusion}

The automatic nerve signal transmission and conscious primacy hypothesis proposes that nerve signals are repeatedly transmitted along a direction. The neural connections in this direction will therefore gradually increase and become strengthened. Furthermore, when responding to stimuli, nerve signals will be automatically transmitted along the strongest neural connections, thereby rapidly generating actions. As soon as stimuli reach conscious awareness, the nerve signals switch to consciousness-guided direction, thereby generating voluntary action. Automatic nerve signal transmission is a natural law in biology and the origin of all behaviors.

\section{Conflicts of Interest}

The author declares no conflicts of interest.

\section{References}

[1] Libet, B., Gleason, C.A., Wright, E.W. and Pearl, D.K. (1983) Time of Conscious Intention to Act in Relation to Onset of Cerebral Activity (Readiness-Potential). The Unconscious Initiation of a Freely Voluntary Act. Brain, 106, 623-642. https://doi.org/10.1093/brain/106.3.623

[2] Galdi, S., Arcuri, L. and Gawronski, B. (2008) Automatic Mental Associations Predict Future Choices of Undecided Decision-Makers. Science, 321, 1100-1102. https://doi.org/10.1126/science.1160769

[3] Soon, C.S., Brass, M., Heinze, H.J. and Haynes, J.D. (2008) Unconscious Determinants of Free Decisions in the Human Brain. Nature Neuroscience, 11, 543-545. https://doi.org/10.1038/nn.2112

[4] Marshall, J.C. and Halligan, P.W. (1988) Blindsight and Insight in Visuo-Spatial 
Neglect. Nature, 336, 766-767. https://doi.org/10.1038/336766a0

[5] Theeuwes, J., Kramer, A.F., Hahn, S. and Irwin, D.E. (1998) Our Eyes Do Not Always Go Where We Want Them to Go: Capture of the Eyes by New Objects. Psychological Science, 9, 379-385. https://doi.org/10.1111/1467-9280.00071

[6] Bode, S., He, A.H., Soon, C.S., Trampel, R., Turner, R. and Haynes, J.D. (2011) Tracking the Unconscious Generation of Free Decisions Using Ultra-High Field fMRI. PLoS ONE, 6, e21612. https://doi.org/10.1371/journal.pone.0021612

[7] Fried, I., Mukamel, R. and Kreiman, G. (2011) Internally Generated Preactivation of Single Neurons in Human Medial Frontal Cortex Predicts Volition. Neuron, 69, 548-562. https://doi.org/10.1016/j.neuron.2010.11.045

[8] Piccolino, M. (1998) Animal Electricity and the Birth of Electrophysiology: The Legacy of Luigi Galvani. Brain Research Bulletin, 46, 381-407.

[9] Schott, G.D. (1993) Penfield's Homunculus: A Note on Cerebral Cartography. Journal of Neurology, Neurosurgery and Psychiatry, 56, 329-333. https://doi.org/10.1136/jnnp.56.4.329

[10] Kandel, E.R. (2006) In Search of Memory: The Emergence of a New Science of Mind. W.W. Norton \& Company, New York, 187-197.

[11] Shadlen, M.N. and Newsome, W.T. (1998) The Variable Discharge of Cortical Neurons: Implications for Connectivity, Computation, and Information Coding. Journal of Neuroscience, 18, 3870-3896.

[12] Burkitt, A.N. (2006) A Review of the Integrate-and-Fire Neuron Model: I. Homogeneous Synaptic Input. Biological Cybernetics, 95, 1-19. https://doi.org/10.1007/s00422-006-0068-6

[13] Midtgaard, J. (1994) Processing of Information from Different Sources: Spatial Synaptic Integration in the Dendrites of Vertebrate CNS Neurons. Trends in Neuroscience, $17,166-173$

[14] Gulledge, A.T., Kampa, B.M. and Stuart, G.J. (2005) Synaptic Integration in Dendritic Trees. Journal of Neurobiology, 64, 75-90. https://doi.org/10.1002/neu.20144

[15] Pinel, J.P.J. (2011) Biopsychology. 8th Edition, Pearson Education, Inc., Boston, 75-98.

[16] Hebb, D.O. (1949) The Organization of Behavior. Wiley \& Sons, New York.

[17] Kleim, J.A., Lussnig, E., Schwarz, E.R., Comery, T.A. and Greenough, W.T. (1996) Synaptogenesis and Fos Expression in the Motor Cortex of the Adult Rat after Motor Skill Learning. Journal of Neuroscience, 16, 4529-4535.

[18] Foster, T.C., Gagne, J. and Massicotte, G. (1996) Mechanism of Altered Synaptic Strength Due to Experience: Relation to Long-Term Potentiation. Brain Research, 736, 243-250.

[19] Sharp, P.E., McNaughton, B.L. and Barnes, C.A. (1985) Enhancement of Hippocampal Field Potentials in Rats Exposed to a Novel, Complex Environment. Brain Research, 339, 361-365.

[20] Kleim, J.A., Vij, K., Ballard, D.H. and Greenough, W.T. (1997) Learning-Dependent Synaptic Modifications in the Cerebellar Cortex of the Adult Rat Persist for at Least Four Weeks. Journal of Neuroscience, 17, 717-721.

[21] Jaśkowski, P. and Slósarek, M. (2007) How Important Is a Prime's Gestalt for Subliminal Priming? Consciousness and Cognition, 16, 485-497.

[22] Schlaghecken, F. and Eimer, M. (2000) A Central-Peripheral Asymmetry in Masked Priming. Perception \& Psychophysics, 62, 1367-1382.

https://doi.org/10.3758/BF03212139 
[23] Kühn, S. and Brass, M. (2009) Retrospective Construction of the Judgement of Free Choice. Consciousness and Cognition, 18, 12-21.

[24] Storbeck, J. and Clore, G.L. (2007) On the Interdependence of Cognition and Emotion. Cognition \& Emotion, 21, 1212-1237. https://doi.org/10.1080/02699930701438020

[25] Lai, V.T., Hagoort, P. and Casasanto, D. (2012) Affective Primacy vs. Cognitive Primacy: Dissolving the Debate. Frontiers in Psychology, 3, 243. https://doi.org/10.3389/fpsyg.2012.00243

[26] Porter, S. and ten Brinke, L. (2008) Reading Between the Lies: Identifying Concealed and Falsified Emotions in Universal Facial Expressions. Psychological Science, 19, 508-514. https://doi.org/10.1111/j.1467-9280.2008.02116.x

[27] Baggott, S., Palermo, R. and Williams, M.A. (2011) Involuntary Facial Expression Processing: Extracting Information from Two Simultaneously Presented Faces. PLoS ONE, 6, e22287. https://doi.org/10.1371/journal.pone.0022287

[28] Dimberg, U., Thunberg, M. and Elmehed, K. (2000) Unconscious Facial Reactions to Emotional Facial Expressions. Psychological Science, 11, 86-89. https://doi.org/10.1111/1467-9280.00221

[29] Lally, P., Wardle, J. and Gardner, B. (2011) Experiences of Habit Formation: A Qualitative Study. Psychology, Health \& Medicine, 16, 484-489. https://doi.org/10.1080/13548506.2011.555774

[30] Tam, L., Bagozzi, R.P. and Spanjol, J. (2010) When Planning Is Not Enough: The Self-Regulatory Effect of Implementation Intentions on Changing Snacking Habits. Health Psychology, 29, 284-292. https://doi.org/10.1037/a0019071

[31] Gratton, L., Povey, R. and Clark-Carter, D. (2007) Promoting Children's Fruit and Vegetable Consumption: Interventions Using the Theory of Planned Behaviour as a Framework. British Journal of Health Psychology, 12, 639-650. https://doi.org/10.1348/135910706X171504

[32] Solms, M. (2004) Freud Returns. Scientific American, 290, 82-88. https://doi.org/10.1038/scientificamerican0504-82

[33] Chessick, R.D. (2010) Returning to Freud. Journal of the American Academy Psychoanalysis and Dynamic Psychiatry, 38, 413-439. https://doi.org/10.1521/jaap.2010.38.3.413

[34] Malinowski, J.E. and Horton, C.L. (2014) Memory Sources of Dreams: the Incorporation of Autobiographical Rather than Episodic Experiences. Journal of Sleep Research, 23, 441-447. https://doi.org/10.1111/jsr.12134

[35] Perrin, F., García-Larrea, L., Mauguière, F. and Bastuji, H. (1999) A Differential Brain Response to the Subject's Own Name Persists during Sleep. Clinical Neurophysiology, 110, 2153-2164. https://doi.org/10.1016/s1388-2457(99)00177-7

[36] Holeckova, I., Fischer, C., Giard, M.H., Delpuech, C. and Morlet, D. (2006) Brain Responses to a Subject's Own Name Uttered by a Familiar Voice. Brain Research, $1082,142-152$. 
Submit or recommend next manuscript to SCIRP and we will provide best service for you:

Accepting pre-submission inquiries through Email, Facebook, LinkedIn, Twitter, etc. A wide selection of journals (inclusive of 9 subjects, more than 200 journals)

Providing 24-hour high-quality service

User-friendly online submission system

Fair and swift peer-review system

Efficient typesetting and proofreading procedure

Display of the result of downloads and visits, as well as the number of cited articles Maximum dissemination of your research work

Submit your manuscript at: http://papersubmission.scirp.org/

Or contact jbbs@scirp.org 